\title{
Association of the MYH9 gene polymorphisms with chronic renal disease secondary to hypertensive nephrosclerosis, in a Caucasian population
}

\author{
Diez-Ojea Beatriz ${ }^{1}$, Marin Rafael ${ }^{2}$, Coto Eliecer $^{3}$, Tavira Beatriz ${ }^{3}$, Fernandez-Vega Francisco ${ }^{4}$, \\ Alvarez Rafael $^{4}$, Fernandez-Fresnedo Gema ${ }^{5}$, Pobes Alfonso ${ }^{6}$, Suarez-Laures Ana ${ }^{7}$, \\ Garcia-Monteavaro Camino ${ }^{8}$, Gorostidi Manuel ${ }^{4}$, Sanchez Emilio $^{4}$, Arias Manuel ${ }^{5}$, \\ Ortega Francisco ${ }^{4}$
}

\footnotetext{
${ }^{1}$ Nephrology Service, Hospital Universitario De Torrevieja-Vinalopo, Alicante, Spain

${ }^{2}$ Nephrology Service, Centro Médico De Asturias, Oviedo (Asturias), Spain

${ }^{3}$ Laboratory of Molecular Genetics, Hospital Universitario Central De Asturias, Oviedo (Asturias), Spain

${ }^{4}$ Nephrology Service, Hospital Universitario Central De Asturias, Oviedo (Asturias), Spain

${ }^{5}$ Nephrology Service, Hospital Universitario Marqués De Valdecilla, Santander (Cantabria), Spain

${ }^{6}$ Nephrology Service, Hospital Valle Del Nalón, Langreo (Asturias), Spain

${ }^{7}$ Nephrology Service, Hospital De Cabueñes, Gijón (Asturias), Spain

${ }^{8}$ Nephrology Service, Hospital San Agustín, Avilés (Asturias), Spain
}

Email address:

beaojea@hotmail.com (B. D. Ojea)

To cite this article:

Diez-Ojea Beatriz, Marin Rafael, Coto Eliecer, Tavira Beatriz, Fernandez-Vega Francisco, Alvarez Rafael, Fernandez-Fresnedo Gema, Pobes Alfonso, Suarez-Laures Ana, Garcia-Monteavaro Camino, Gorostidi Manuel, Sanchez Emilio, Arias Manuel, Ortega Francisco. Association of the MYH9 Gene Polymorphisms with Chronic Renal Disease Secondary to Hypertensive Nephrosclerosis, in a Caucasian Population. American Journal of Internal Medicine. Vol. 2, No. 6, 2014, pp. 95-101. doi: 10.11648/j.ajim.20140206.11

\begin{abstract}
Background: Hypertensive nephrosclerosis (HN) is a chronic kidney disease (CKD) associated to essential hypertension, but their causal relationship is controversial. New evidence suggests that $M Y H 9$ gene alterations are associated with $\mathrm{HN}$ in African Americans. The aim of this study is to investigate the role of this gene in Spanish Caucasians. Methods: We compare high-risk MYH9 variants of patients with $\mathrm{HN}$ recruited according to standard clinical criteria (CKD stages 3-5), with essential hypertensives without renal disease (estimated glomerular filtration rate (eGFR) $>60 \mathrm{ml} / \mathrm{min} / 1,73 \mathrm{~m}^{2}$ and albuminuria $<300 \mathrm{mg} / \mathrm{g}$ creatinine), and also CKD patients with $\mathrm{HN}$ and progressive impairment of renal function with those who were stable. Diabetics were excluded. Results: A blood sample was obtained for genetic study of 238 patients with HN-CKD and 233 hypertensive controls. The rs3752462-T and rs4821480-T (risk alleles for CKD) were more frequent in the CKD group, but without significant difference. We found no differences for these SPNs with blood pressure, creatinine, albuminuria or renal disease progression. Conclusions: The effect of two common MYH9 single nucleotide polymorphisms (SPNs) on the development of CKD secondary to HN in our Spanish Caucasian population is low or zero; in any case less than that found in other, mainly African Americans.
\end{abstract}

Keywords: Chronic Kidney Disease, Essential Hypertension, Hypertensive Nephrosclerosis, MYH9 Polymorphisms

\section{Introduction}

The term Hypertensive Nephrosclerosis (HN) is usually used for kidney disease which complicates arterial hypertension and primarily affects the preglomerular microvasculature. In practice, it is an entity with vague clinical profiles that groups hypertensive patients with chronic kidney disease (CKD) without other identifiable causes of disease ${ }^{1}$. In the USA, Europe and Spain, vascular kidney disease is the second most common cause of Endstage renal disease $(E S R D)^{2}$. Compared with early glomerular or diabetic kidney diseases, the progression of kidney failure is slow in most cases, especially in Caucasians. Renal function can remain stable for years if hypertension is 
well controlled. However, in a few cases the disease progresses to $\mathrm{ESRD}^{3}$.

The lack of correlation between the degree of hypertension control and the prevention of disease progression, from a clinical and histological standpoint suggests that this process may be an intrinsic and early renal disease ${ }^{4}$.

$\mathrm{HN}$ genetic studies have been conducted primarily in African Americans because the disease is more frequent and aggressive in black patients ${ }^{5}$. Recently published studies have led to a new approach to the pathogenesis of HN among African Americans. They reveal a close relationship between the presence of ESRD secondary to HN in non-diabetics, and some single nucleotide polymorphisms (SNPs)/haplotipes of the nonmuscle myosin heavy chain 9 gene (MYH9 gene $)^{6-8}$, and in the apolipoprotein L1 gene (APOL1 gene $)^{9-11}$. Both genes are located on chromosome 22 , and these risk alleles are much less common in Caucasians.

Only a few studies have been conducted in Caucasians on this subject to verify whether these or other SNPs might be involved in the development of $\mathrm{HN}^{12-16}$.

Since we previously observed $\mathrm{HN}$ is a CKD that rarely progresses in Caucasians, we designed the present study to determine if any of the MYH9 gene SNPs are linked in this race with the association of essential hypertension and $\mathrm{HN}$ and with progression of the renal disease.

\section{Materials and Methods}

\subsection{Study Design and Data Collection ${ }^{17}$}

This is a retrospective case-control study to evaluate the association between SNPs and HN, comparing HN patients with hypertensive patients without kidney disease (control group); besides, patients with $\mathrm{HN}$ and progressive increasing of renal function impairment were compared with those who were stable.

All individuals were non-related Caucasians from the Spanish regions Asturias and Cantabria (Northern Spain, total population one and a half million inhabitants).

The patients were selected between October 2009 and October 2011. They had the glomerular filtration rate (eGFR) $<60 \mathrm{ml} / \mathrm{min} / 1.73 \mathrm{~m}^{2}$ (measured by the MDRD formula ${ }^{18}$ ) in stages $3-5$, attributed to $\mathrm{HN}$ according to standard clinical criteria (blood pressure $\geq 140 / 90 \mathrm{mmHg}$ or on antihypertensive medication, Creatinine $\geq 1.5 \mathrm{mg} / \mathrm{dl}$ in men or $\geq 1.4 \mathrm{mg} / \mathrm{dl}$ in women, eGFR $<60 \mathrm{ml} / \mathrm{min} / 1.73 \mathrm{~m}^{2}$, proteinuria $\leq 3.5 \mathrm{~g} / 24$ hours, and no other identifiable causes of kidney disease including ischaemic kidney disease), except in cases in which there was histological confirmation of NH. Diagnosis of CKD of unknown origin was not valid. Diabetics, morbid obesity, one-kidney, and secondary, renovascular or accelerated hypertensive patients (opthalmoscopy grade III or IV) were excluded. Patients with CKD stage 5, on dialysis or to undergo kidney transplant, were admitted, provided that developmental data were available from their first contact with the hospital. The start of renal-replacement therapy (RRT) also marked the end of the follow-up.

The hypertensive control group was from the same geographic area. Patients with essential hypertension were included with eGFR $>60 \mathrm{ml} / \mathrm{min} / 1.73 \quad \mathrm{~m}^{2}$ and microalbuminuria $<300 \mathrm{mg} / \mathrm{g}$ creatinine.

Progression of CKD was defined as doubling of the serum creatinine level from baseline, a reduction of $50 \%$ in the eGFR or $25 \mathrm{ml} / \mathrm{min} / 1.73 \mathrm{~m}^{2}$ from baseline, or ESRD onset (defined by the need for RRT).

There were two sections of the study, one for the first hospital visit and one for the last ("current visit"). Clinical data included socio-demographic variables, comorbidity and cardiovascular risk, medical treatment and physical examination. Analytical data included renal function by serum creatinine, eGFR, creatinine clearance, and also glucose, uric acid, cholesterol and calcium-phosphorus metabolism and albuminuria in a single sample of urine (albumin/creatinine $\mathrm{mg} / \mathrm{g}$ ) and proteinuria in 24-hours urine collections. Furthermore, imaging techniques (ultrasound, CT angiography, MR angiography or arteriography) and renal biopsy diagnosis were also included in the study of some patients.

This study was approved by the Research Ethics Committee of the Regional Clinical Research of Asturias and all the participants signed an informed consent.

\subsection{Laboratory Analysis}

Routine fasting blood tests were performed by the usual methods in the laboratory of each hospital. Blood samples for genetic studies were submitted to the Laboratory of Molecular Genetics, Hospital Universitario Central de Asturias. DNA was obtained from leukocytes from $10 \mathrm{ml}$ of EDTA anticoagulated peripheral blood. The samples will be stored for 5 years.

Major MYH9 SNPs to be identified were those which showed an association with hypertensive nephrosclerosis in non-diabetics (rs4821480 and rs3752462, which tagged E-1 haplotype). DNA was obtained from $5 \mathrm{ml}$ of blood leukocytes, and the SNPs were genotyped through a Polymerase Chain Reaction-restriction fragment length polymorphism (PCR-RFLP) method. Briefly, for the rs3752462 a 421 bp fragment was amplified with primers CCAGGAGCATCCGGGCTCTA (forward) and CACCTCCACAACCAACACAGAGCT (reverse). After digestion with RsaI, reactions were electrophoresed on $3 \%$ agarose gels and the two alleles were visualized as bands of $243+178$ bp (C allele) and $243+92+86$ bp (T allele). For the rs4821480 the DNA was PCR amplified with primers CCGCTGGGCAGGGGTGTT (forward) and TCTTCTGTG AGGTTGGT GGTG (reverse), followed by digestion with DraI and electrophoresis on agarose gels to visualize the two alleles of $537 \mathrm{bp}$ ( $\mathrm{G}$ allele) and $322+215 \mathrm{bp}$ (T allele). To confirm the accuracy of this genotyping method, PCR fragments from several individuals with each of the RFLPgenotypes were sequenced using BigDye chemistry in an ABI3130 automated system (Applied Biosystems). 


\subsection{Statistical Analysis}

A descriptive analysis of continuous variables was done, giving the mean, median, standard deviation and range, and in case of discrete variables, the frequency distribution and percentages. $95 \%$ confidence intervals were calculated when necessary. The association between qualitative variables has been assessed with the Chi-square or Fisher's exact test and quantitative variables using parametric tests (t-test, Pearson correlation coefficient and ANOVA). When a normal distribution could not be assumed, non-parametric tests were

used, such as Mann-Whitney $U$ or Kruskal-Wallis. Comparison was considered statistically significant when Pvalue $<0.05$. We have used for multivariate analysis a decision tree with CHAID method, based on Chi-square test, and the multivariate regression analysis was performed to determine the independent effect of the quantitative variables. Statistical analysis of the study has been conducted with SPSS for Windows, version 17.0 (SPSS, Chicago, IL).

\section{Results}

\section{ESSENCIAL HYPERTENSIVES RECRUITED ( $n=759)$}

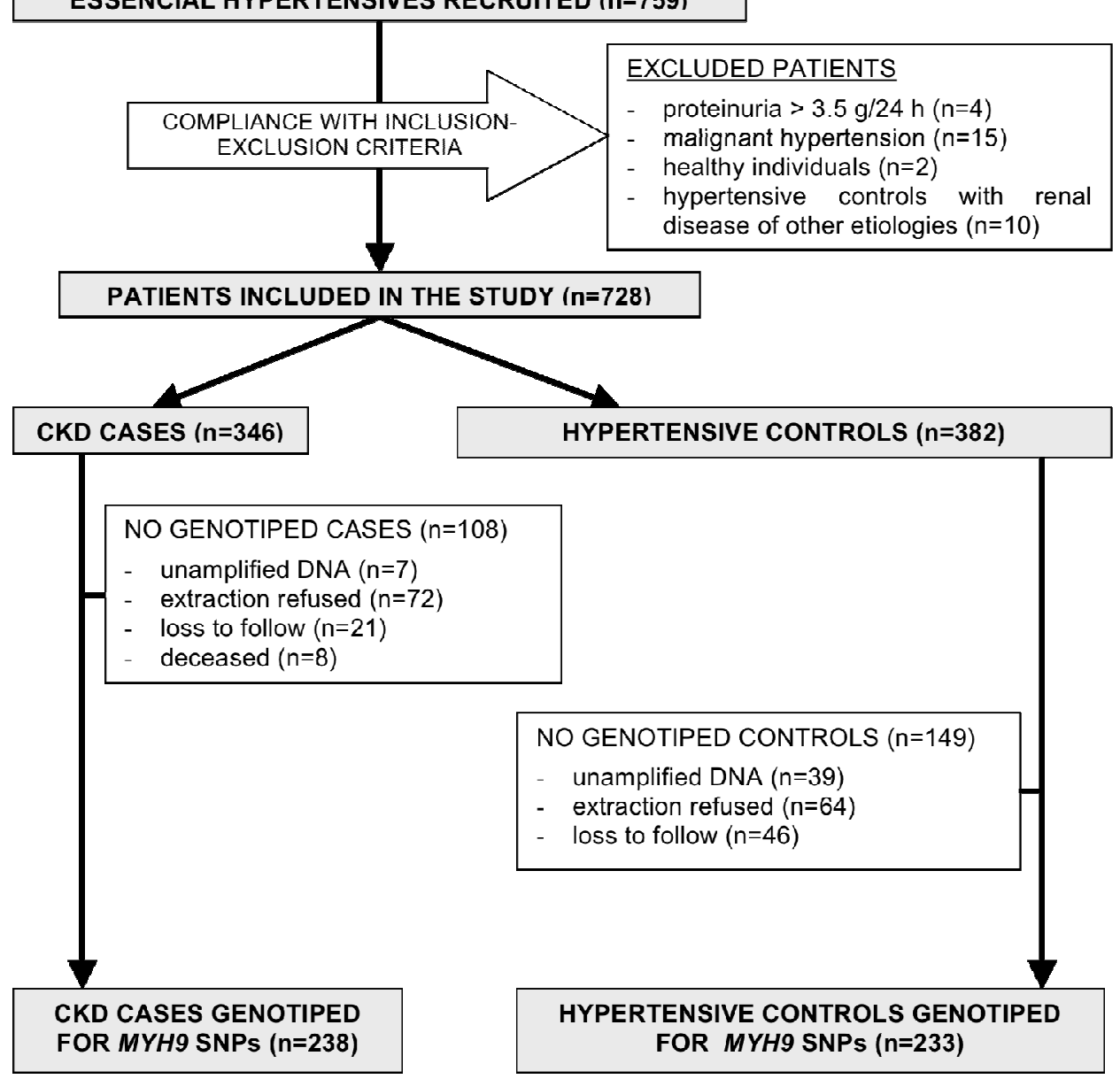

Figure 1. Patients included in the study.

728 Caucasian subjects were finally included in the study of the 759 eligible. We have analyzed 346 cases with CKD and clinical diagnosis of $\mathrm{HN}$, and 382 hypertensive controls without renal disease. Patient refuse, lost to follow up or technical procedure problems were the main causes of genotype lacking. Finally, 238 CKD cases and 233 hypertensive controls were genotyped for rs3752462 and rs4821480 MYH9 SNPs (Figure 1). No statistically significant differences were found among individuals who were successfully genotyped and those who were not. Median follow-up was 41.8 months, without difference between the groups.

Table 1, summarizes the main clinical characteristics in the whole study and the genotype frequencies for the two MYH9 SNPs. No statistically significant differences were found for both SNPs among groups (CKD cases and hypertensive controls), although there was a trend (not significant) for the risk genotype $\mathrm{T}(\mathrm{CT}+\mathrm{TT})$ of rs3752462 SNP to develop CKD secondary to $\mathrm{NH}(0.52$ vs. 0.48 ; $\mathrm{OR}=1.10 ; 95 \% \mathrm{CI} 0.80-1.52$; $\mathrm{p}=0.54$ between CKD group and hypertensives), and for a power of $80 \%$ at a $\mathrm{p}=0.05$, a total of 2,452 individuals in both groups should be required to exclude an association to 
CKD. Nor have we found statistically significant differences for these SPNs with blood pressure, serum creatinine, or baseline albuminuria.

Over a half of the 238 CKD cases (49.2\%) had progression of CKD, according to our definition, but most of the patients who reached ESRD were recruited when they were already on RRT (dialysis or transplantation), and their clinical data were collected retrospectively. If we do not consider these historical patients, 141 CKD cases were selected in the clinic and were followed up prospectively.
Only 20 patiens $(14.2 \%)$ in this subgroup suffered progressive increasing of renal function impairment (9 started RRT, 8 had halving eGFR above baseline and 3 had a $25 \mathrm{ml} / \mathrm{min} / 1.73 \mathrm{~m}^{2}$ eGFR reduction). Anyway, no statistically significant differences were found between MYH9 SNPs and renal disease progression (Table 2).

In the CKD clinical progession markers study, serum creatinine, proteinuria and hemoglobin baseline, and a higher age, were significantly associated with renal function impairment in the multivariate analysis.

Table 1. Main values in the whole study population and the genotype frequencies for the two MYH9 SNPS.

\begin{tabular}{|c|c|c|c|}
\hline Main characteristics & CKD-cases $(n=238)$ & Hypertensive controls $(n=233)$ & p values \\
\hline$\%$ Male & $0.76(238)$ & $0.52(233)$ & $<0.001$ \\
\hline Age (years) & $71.2 \pm 8.3(238)$ & $61.4 \pm 10.9(233)$ & $<0.001$ \\
\hline Body-mass index (kg/m2) & $28.7 \pm 4.2(199)$ & $29.4 \pm 4.7(231)$ & 0.077 \\
\hline \% Hypercholesterolemics & $0.76(214)$ & $0.76(233)$ & 0.955 \\
\hline$\%$ Smokers & $0.21(215)$ & $0.13(233)$ & $<0.001$ \\
\hline$\%$ Cardiovascular comorbiditya & $0.60(235)$ & $0.39(233)$ & $<0.001$ \\
\hline Systolic blood pressure (mmHg) & $151.2 \pm 24.9(214)$ & $147.5 \pm 20.7(232)$ & 0.090 \\
\hline Pulse pressure (mmHg) & $69.6 \pm 20.8(214)$ & $60.7 \pm 17.9(232)$ & $<0.001$ \\
\hline Serum creatinine $(\mathrm{mg} / \mathrm{dl})$ & $4.00 \pm 2.88(209)$ & $0.85 \pm 0.17(233)$ & \\
\hline eGFR $(\mathrm{ml} / \mathrm{min} / 1.73 \mathrm{~m} 2) \mathrm{b}$ & $25.81 \pm 17.05(209)$ & $88.65 \pm 17.08(233)$ & \\
\hline Albuminuria ratio $(\mathrm{mg} / \mathrm{g}) \mathrm{c}$ & $254.8 \pm 515.7(118)$ & $10.59 \pm 19.70(224)$ & \\
\hline $\mathrm{LDL}(\mathrm{mg} / \mathrm{dl})$ & $101.7 \pm 35.0(181)$ & $120.8 \pm 32.2(230)$ & $<0.001$ \\
\hline \multicolumn{4}{|l|}{ MYH9 rs3752462 } \\
\hline $\mathrm{CC}$ & $110 / 230(0.48)$ & $122 / 233(0.52)$ & \\
\hline TT & $31 / 230(0.13)$ & $26 / 233(0.11)$ & 0.251 \\
\hline $\mathrm{C}$ & $309(0.67)$ & $329(0.71)$ & \multirow{2}{*}{0.474} \\
\hline $\mathrm{T}$ & $151(0.33)$ & $137(0.29)$ & \\
\hline \multicolumn{4}{|l|}{ MYH9 rs4821480 } \\
\hline TT & $190 / 208(0.91)$ & $204 / 229(0.89)$ & \multirow{4}{*}{0.172} \\
\hline TG & $18 / 208(0.09)$ & $22 / 229(0.10)$ & \\
\hline GG & $0 / 208(0.00)$ & $3 / 229(0.01)$ & \\
\hline $\mathrm{T}$ & $398(0.96)$ & $430(0.94)$ & \\
\hline G & $18(0.04)$ & $28(0.06)$ & \multirow[t]{2}{*}{0.449} \\
\hline $\mathrm{G}$ & $18(0.04)$ & $28(0.06)$ & \\
\hline
\end{tabular}

${ }^{\text {a }}$ Presence of cardiovascular disease (ischemic heart disease, heart failure, atrial fibrillation, valvular degeneration, stroke, peripheral arterial disease)

${ }^{\mathrm{b}} \mathrm{eGFR}(\mathrm{ml} / \mathrm{min} . / 1.73 \mathrm{~m} 2)=186 \times[\text { plasma creatinine }(\mathrm{mg} / \mathrm{dl})]^{-1,154} \times(\mathrm{age})^{-0,203} \times(0.742$ if female $) \times(1.212$ if black $)$.

${ }^{\mathrm{c}}$ albumin/creatinine ratio.

Demografic and analytical data are from the last visit, or before the start of the RRT.

Table 2. Relationship between MYH9 SPNs and renal disease progression.

\begin{tabular}{lll}
\hline & Progression of CDK & No progression of CDK \\
\hline MYH9 rs3752462 & $58 / 112(0.52)$ & \\
CC & $40 / 112(0.36)$ & $52 / 118(0.44)$ \\
CT & $14 / 112(0.12)$ & $49 / 118(0.42)$ \\
TT & & $17 / 118(0.14)$ \\
MYH9 rs4821480 & $82 / 87(0.94)$ & \\
TT & $5 / 87(0.06)$ & $108 / 121(0.89)$ \\
TG & $0 / 87(0.00)$ & $13 / 121(0.11)$ \\
GG & & $0 / 121(0.01)$ \\
\hline
\end{tabular}

\section{Discussion}

Our results have shown no relationship between $\mathrm{HN}$ and MYH9 SNPs in Spanish Caucasian subjects, as just previously discussed, or at least our study suggests that the effect of MYHO SNPs in our population is less than that found in other, primarily in African Americans.
Due to the very low frequency of the risk allele in our population, to exclude an association to CKD a much large number of individuals should be required in both groups. The sample size required to confirm this effect is the usual in markers with a lower OR, and we believe that our study has an acceptable size to work with most multivariate diseaseassociated SNPs. Moreover, the frequency of these SNPs in the population of Asturias and Cantabria is similar to that 
expected to be found in Caucasians. Therefore, we can not rule that the MYH9 SNPs play a role in the risk for $\mathrm{NH}$, although this effect is likely to have a low value, in any case less than that found in African Americans.

The importance of our work is that it has done in Caucasians. Previous studies existing in literature are in almost all cases of African Americans ${ }^{6-11}$. In there works, the nephrosclerosis sample size, between 200 and 700 individuals, is slightly higher than ours, but to try to show association with a much more prevalent gene in this population. Only in the important study of Genovese et al. with the APOL1 gene, the cohort of nephrosclerosis ESRD patients reaches 1,030 individuals $^{9}$. Also, these studies have significant methodological limitations: only Lipkowitz et al. and Parsa et al. worked with patients with $\mathrm{NH}$, the other publications use diverse etiology CKD and differences in disease severity (CKD and ESRD). Moreover, the few publications in Caucasians collect data from population studies rather than patients with $\mathrm{HN}$ secondary $\mathrm{CKD}^{12-16}$. Our group has shown an independent relationship between the same polymorphism and the presence of an eGFR $<60$ $\mathrm{ml} / \mathrm{min} / 1,72 \mathrm{~m}^{2}$ in a general population sample without previously known renal disease ${ }^{16}$, but the work presented is the only one conducted in Caucasians with $\mathrm{NH}$ select population by strict clinical criteria to date.

Recently, it has been shown that two SNPs in the apolipoprotein L1 nearby gene (APOL1 gene), located on the same chromosome (rs73885319 and rs60910145, haplotype G1) and one deletion (rs71785323, haplotype G2) were strongly linked to CKD/ESRD among African Americans ${ }^{9,11}$. APOL1 is a gene that protects against Trypanosoma brucei rhodesiense, responsible for the "Sleeping Sickness", and the risk alleles are very rare or absent among Caucasians ${ }^{19}$. Although these APOL1 SNPs could explain part of the effect previously attributed to $M Y H 9$ among individuals of African ascent, it has been shown that MYH9 SNPs may have an independent role risk of developing CKD. Probably both genes are likely to be involved, because $A P O L 1$ is not expressed in Caucasians ${ }^{10}$.

MYH9 gene encodes a myosin heavy chain protein expressed in glomerular podocytes. The role of podocyte loss and dysfunction has been reported as part of disease development ${ }^{20}$. In favor of the importance of $M Y H 9$ gene SNPs, stresses that its presence in podocyte dysfunction is more evident than the $A P O L 1$, and is expressed in other populations, not only in those of African descent ${ }^{21}$.

A bold new hypothesis on this issue states that SNPs of these gene would be markers of various kidney diseases that fall into one histological group, focal segmental glomerulosclerosis (FSGS), and any of its variants, such as $\mathrm{HN}$, which would be a primary kidney disease with idiopathic FSGS and with the collapse observed in $\mathrm{HIV}^{22}$. Lastly, there are some rare entities associated with mutations of that gene, which are transmitted by autosomal dominant inheritance, known as MYH9-related disorders ${ }^{23}$. Evidence linking these SNPs to ESRD associated with diabetes is equivocal and will require additional investigation ${ }^{11}$.
However, since the frequency of MYH9 risk alleles in individuals of Caucasian origin is very low, and $\mathrm{NH}$ has a more aggressive behavior in African Americans, this probably is not the same type of kidney disease, and the role of these genes is not vital. Furthermore, they have been shown mainly associated with aggressive variants of the disease, ie, patients who reach ESRD ${ }^{15}$, and it seems that in patients with moderate CKD (as our sample) the risk may be lower ${ }^{10}$. Probably in genetically predisposed patients, other unknown factors (environmental or interaction with other genes) can cause disease ${ }^{19}$.

In our clinical substudy, the main clinical markers of progression of CKD were baseline proteinuria and creatinine, consistent with literature data ${ }^{24}$. Usually it is considered that $\mathrm{NH}$ usually presents with proteinuria less than $1.5 \mathrm{~g} / 24$ hours, although we know that it can be variable, so we decided to include patients with non-nephrotic proteinuria. Of course, cases diagnosed by renal biopsy $(4.3 \%)$ were those with higher proteinuria, probably because other nephropathies were suspected. Proteinuria seems to be constituted as a phenotypic marker for progressors patients ${ }^{25}$.

The originality of our study lies in the careful selection of Caucasian patients with $\mathrm{HN}$, to rule out genetic association of these SNPs with the development of the disease.

The main methodological limitation of our work, like in most of studies, is the absence of histological confirmation of clinical diagnosis, although its indication is an ethical dilemma, given the low therapeutic profitability. However, despite the absence of renal biopsy, the main strength of our work is the strict selection of participants with nephrosclerosis by clinical criteria, such as the AASK study in African American individuals ${ }^{10,11}$.

Although selection attempted paired cases and controls, age and sex-matched, finally cases are proportionately more males (consistent with the epidemiology of CKD), and older than hypertensive controls. However, this limitation is common to AASK study that analyzes MYH9 and APOL1 $\mathrm{SNPs}^{10}$. It is difficult to determine if those years apart influence the genetic risk. In addition, not all patients provided DNA samples or were successfully genotyped, raising the potential for bias.

Other limitations are those related to a retrospective study design, together with the lack of a centralised laboratory for routine analysis, because the data are historic. HN cases that maintain periodic visits over a variable time period and patients that have progressed to ESRD are included. In this respect, we have significant selection bias when calculating the percentage of patients whose disease has progressed, since many have been obtained from dialysis and transplant units. We have probably not been able to draw clear conclusions about disease progression but, despite these limitations, we believe this kind of study will be interesting because there are very few publications to date on this topic.

The search for genetic markers of risk, such as the detection of MYH9 SNPs, is important for several reasons, such as identifying patients predisposed to the development of nephropathy, early therapeutic intervention and identifying 
new potential targets for treatment.

The implications of recent findings in the understanding of the pathogenesis of the disease are vital, and represent a revolution to current theories. It seems clear that mild to moderate hypertension is not the cause of the high prevalence of ESRD in blacks ${ }^{26}$, and in Caucasian individuals frequency is lower because the progression is not dependent on genetic factors, but on poor control of BP and other cardiovascular risk factors, as we have shown in our research.

It seems necessary to design prospective genetic studies to determine the relationship with this gene, both in terms of diagnosis and the possibility that it could be a progression marker.

\section{Contributorship}

All the authors contributed to this work by recruiting the population cohort (B.D., R.M., F.F., R.A., G.F., A.P., A.S., C.G., M.G., E. S., M. A., F. O.) or performing the genetic analysis (B.T., E.C.).

The results presented in this paper have not been published previously in whole or in part, except as an abstract presented at the Annual Meetings 2012 of the Spanish Society of Nephrology (S.E.N).

\section{Acknowledgments}

We are grateful to all patients who selflessly participated in this study.

This work was supported by grant Red de Investigación Renal-REDINREN from the Instituto Carlos III, and with funds from the Fundación Renal Iñigo Álvarez de Toledo (Iñigo Álvarez de Toledo Kidney Foundation) in the Molecular Genetics Group.

\section{References}

[1] Luke R. Hypertensive nephrosclerosis: pathogenesis and prevalence. Essential hypertension is an important cause of end-stage renal disease. Nephrol Dial Transplant 1999;14:2271-8.

[2] Stengel B, Billon S, Van Dijk PC, et al. Trends in the incidence of renal replacement therapy for end-stage renal disease in Europe, 1990-1999. Nephrol Dial Transplant 2003;18:1824-33.

[3] Siewert-Delle A, Ljungman S, Andersson OK, Wilhelmsen L. Does treated primary hypertension lead to end-stage renal disease? A 20-year follow-up of the Primary Prevention Study in Göteborg, Sweden. Nephrol Dial Transplant 1998;13:308490 .

[4] Skorecki KL, Wasser WG. Hypertension-misattributed kidney disease in African Americans. Kidney Int 2013;83:6-9.

[5] Marcantoni C, Ma LJ, Federspiel C, Fogo AB. Hypertensive nephrosclerosis in African Americans versus Caucasians. Kidney Int 2002;62:172-80.

[6] Kao WH, Klag MJ, Meoni LA, et al. MYH9 is associated with nondiabetic end-stage renal disease in African Americans. Nat Genet 2008;40:1185-92.

[7] Kopp JB, Smith MW, Nelson GW, et al. MYH9 is a majoreffect risk gene for focal segmental glomerulosclerosis. Nat Genet 2008;40:1175-84.

[8] Freedman BI, Hicks PJ, Bostrom MA, et al. Polymorphisms in the non-muscle myosin heavy chain 9 gene (MYH9) are strongly associated with end-stage renal disease historically attributed to hypertension in African Americans. Kidney Int 2009;75:736-45.

[9] Genovese G, Friedman DJ, Ross MD, et al. Association of trypanolytic ApoL1 variants with kidney disease in African Americans. Science 2010;329:841-5.

[10] Lipkowitz MS, Freedman BI, Langefeld CD, et al. Apolipoprotein L1 gene variants associate with hypertensionattributed nephropathy and the rate of kidney function decline in African Americans. Kidney Int 2013;83:114-20.

[11] Parsa A, Kao WHL, Xie D, et al. APOL1 risk variants, race, and progression of chronic kidney disease. N Engl J Med 2013 Nov 9. [Epub ahead of print].

[12] Pattaro C, Aulchenko YS, Isaacs A, et al. Genome- wide linkage analysis of serum creatinine in three isolated European populations. Kidney Int 2009;76:297-306.

[13] O'Seaghdha CM, Parekh RS, Hwang SJ, et al. The MYH9/APOL1 region and chronic kidney disease in European-Americans. Hum Mol Genet 2011;20:2450-6. .

[14] Cooke JN, Bostrom MA, Hicks PJ, et al. Polymorphisms in MYH9 are associated with diabetic nephropathy in European Americans. Nephrol Dial Transplant 2012;27:1505-11.

[15] McKnight AJ, Duffy S, Fogarty DG, Maxwell AP. Association of MYH9/APOL1 with chronic kidney disease in a UK population. Nephrol Dial Transplant 2012(27):3660.

[16] Tavira B, Coto E, Gómez J, et al. Association between a MYH9 polymorphism (rs3752462) and renal function in the Spanish RENASTUR cohort. Gene 2013;520(1):73-6.

[17] Diez Ojea B, Marin R, Coto E, et al. Clinical and genetic bases of Hipertensive Nephrosclerosis. NEFROSEN Study. Nefrología 2010;30(6):687-97.

[18] Levey AS, Bosch JP, Lewis JB, Greene T, Rogers N, Roth D. A more accurate method to estimate glomerular filtration rate from serum creatinine: a new prediction equation. Modification of Diet in Renal Disease Study Group. Ann Intern Med 1999;130, 461-70.

[19] Freedman BI, Langefeld CD. The new era of APOL1associated glomerulosclerosis. Nephrol Dial Transplant 2012;27:1288-91.

[20] Wang G, Lai FM, Kwan BC, et al. Podocyte Loss in Human Hypertensive Nephrosclerosis. Am J Hypertens 2009;22:300-6.

[21] Freedman BI, Langefeld CD, Turner J, et al. Association of APOL1 variants with mild kidney disease in the first-degree relatives of African American patients with non-diabetic endstage renal disease. Kidney Int 2012;82:805-11.

[22] Murea M, Freedman BI. Essential hypertension and risk of nephropathy: a reappraisal. Curr Opin Nephrol Hypertens 2010;19:235-41. 
[23] Singh N, Nainani N, Arora P, Venuto RC. CKD in MYH9Related Disorders. Am J Kidney Dis 2009;54:732-40.

[24] Vikse BE, Aasarød K, Bostad L, Iversen BM. Clinical prognostic factors in biopsy- proven benign nephrosclerosis. Nephrol Dial Transplant 2003;18:517-23.
[25] Jafar TH, Stark PC, Schmid $\mathrm{CH}$, et al. Proteinuria as a modifiable risk factor for the progression of non-diabetic renal disease. Kidney Int 2001;60:1131-40.

[26] Freedman BI, Murea M. Target organ damage in African American hypertension: role of APOL1. Curr Hypertens Rep 2012;14:21-8. 